\title{
Total spondylectomy for primary tumor of the thoracolumbar spine
}

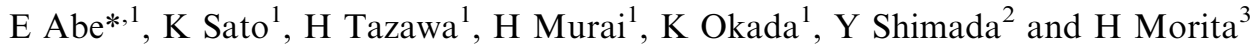 \\ ${ }^{1}$ Department of Orthopedic Surgery, Akita University School of Medicine, Akita, Japan; ${ }^{2}$ Division of Rehabilitation, \\ Akita University School of Medicine, Akita, Japan; ${ }^{3}$ Department of Orthopedic Surgery, Akita Kumiai Hospital, \\ Akita, Japan
}

\begin{abstract}
Study Design: Six patients with primary malignant tumor of the thoracolumbar spine who underwent total spondylectomy (TS) by en bloc resection were reviewed retrospectively.

Objectives: To report surgical technique and preliminary results of TS and to evaluate its oncological curability.

Setting: Japan.

Methods: Six patients were treated by TS by en bloc resection of the vertebral tumor. TS through a posterior approach was performed in three cases (T1 osteosarcoma, L1 osteosarcoma and L1 chordoma) and in the others through a single stage anterior and posterior combined approach (T6-8 recurrent giant cell tumor, L4 chordoma and L5 giant cell tumor). Surgical margins of the specimens were evaluated histologically. All patients were followed, and their status was evaluated by clinical and imaging studies.

Results: There were no complications related to surgery. Programmed sacrifice of nerve roots were performed in three cases for oncologic excision. A wide surgical margin was achieved in one case, a marginal one in four, and an intralesional margin in one. Five patients were alive without evidence of tumor and one was alive with disease at follow-up evaluation after 2.0 4.8 years. Local recurrence was found in one case of T1 osteosarcoma with an intralesional margin.
\end{abstract}

Conclusions: These preliminary results suggested that TS is an effective procedure in control of local recurrence with acceptable complications.

Spinal Cord (2000) 38, 146-152

Keywords: primary vertebral tumor; total spondylectomy; en bloc resection

\section{Case reports}

\section{Case 6}

A 53-year-old man with lower back pain, bilateral thigh pain lasting 2 years, and progressive motor weakness for 4 months was referred to our clinic. Plain radiographs did not reveal any abnormal findings, but MR images demonstrated an L4 vertebral tumor that extended into the epidural space (Figure 1A,B). The condition was classified as IA by Enneking's staging system and 5-8 and BCD by the WBB staging system. Total en bloc laminectomy of the L4 and open biopsy were performed by osteotomy at the pars interarticularis. Histological examination revealed chordoma. Consecutively, the patient underwent en bloc resection of the L4 vertebral tumor through the same posterior approach. Although we tried to roll out the completely isolated L4 vertebra between the L3 and L4 roots, we failed to do it and caused dural tear on the bilateral sides. Accordingly, after posterior stabilization by

*Correspondence: E Abe, Department of Orthopedic Surgery, Akita University School of Medicine, Hondo 1-1-1, Akita 010-8543, Japan pedicle screw fixation system and posterior bone grafting between the adjacent laminae, the patient was placed in an oblique supine position and the isolated L4 vertebra was pulled out by the anterior left retroperitoneal approach (Figure 1C). Anterior spinal reconstruction was performed with a titanium mesh cylinder, autografts and Zielke's instrumentation. At the 2-year follow-up examination, the patient complained of no symptoms, was completely recovered neurologically, and was disease-free oncologically (Figure 1D).

\section{Methods}

The six patients were three females and three males aged from 18-61 (average, 41) years old at the time of surgery. The diagnosis was osteosarcoma in two cases, chordoma in two cases and giant cell tumor in two cases (Table 1). Four patients had preoperative neurologic complications. Five patients had singlelevel lesions, and one had three-vertebral involvement. The tumors included a recurrent giant cell tumor 18 months after piecemeal total spondylectomy and an osteosarcoma 1 month after decompressive laminect- 

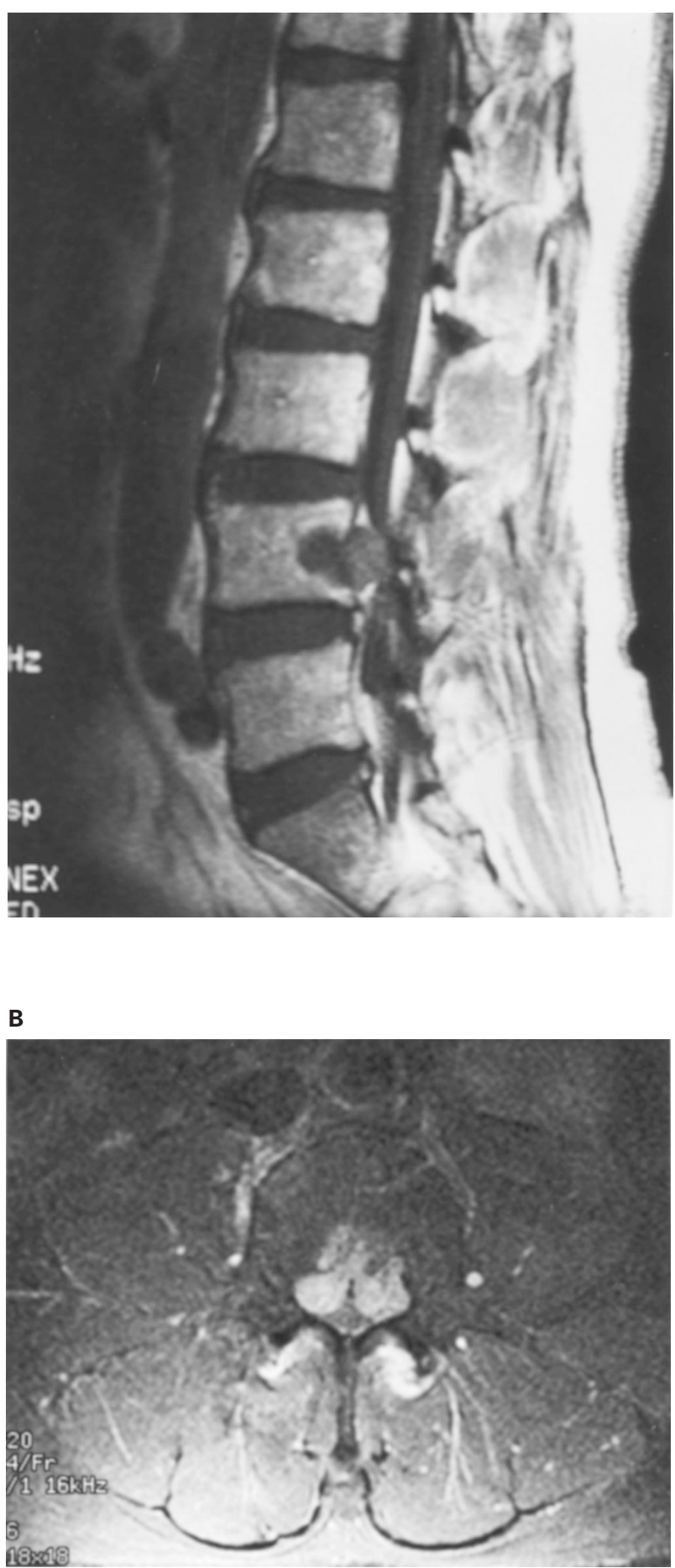

C

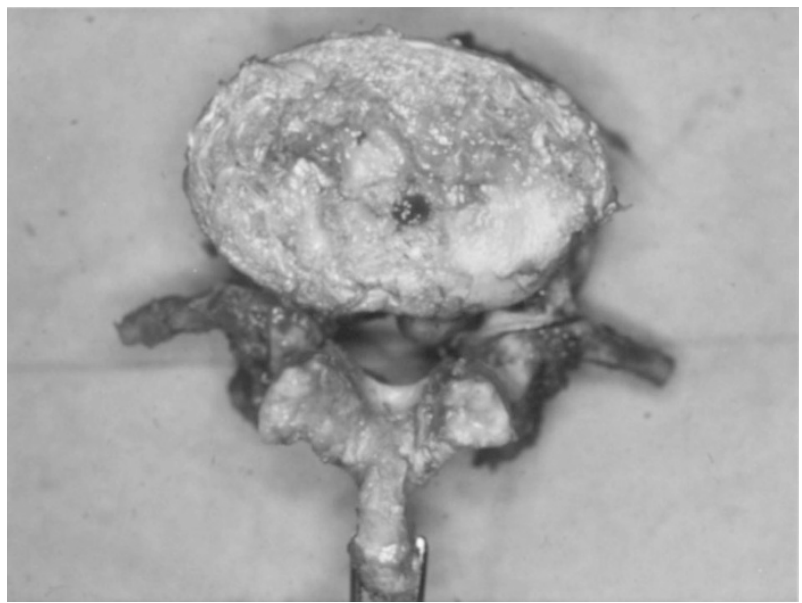

D

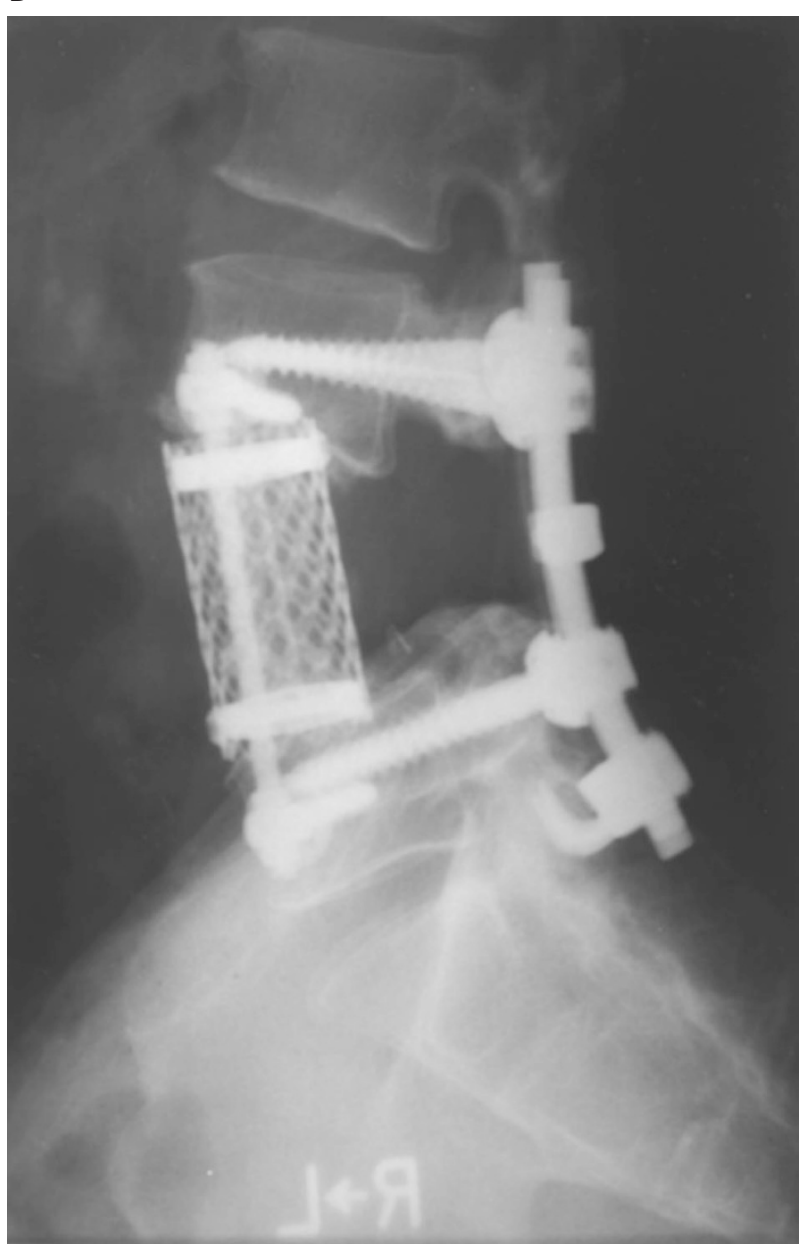

Figure 1 Case 6, chordoma at L4. MR image shows vertebral tumor in the posterior portion in the L4 vertebral body and epidural extension (A) Gd-DTPA enhanced T1-weighted image in the sagittal plane showing a hypoenhanced tumor, (B) T2weighted image in the transaxial plane at L4 showing a high signal-intensity tumor). (C) The transaxial view of the resected L4 vertebra, which was osteotomized at the bilateral pedicles and the interarticular part. (D) Radiograph at 2-year followup showed satisfactory stabilization with pedicle screw fixation, Zielke's instrumentation, titanium mesh cylinder and bone graft 
omy with incomplete intralesional excision of the tumor. The level of the lesions ranged from T1 to L5 (T1, T6-8, L1, L1, L4, L5). Examination showed no distinct evidence of distant metastasis in any patients at the time of surgery. The spinal tumors were classified according to Enneking's staging system, ${ }^{1}$ and also classified according to the WWB staging system, ${ }^{2}$ in which the vertebra in the transaxial plane was divided into 12 radiating zones (numbered $1-12$ in clockwise order) and five layers (A-E from the paravertebral to the dural involvement), and each tumor is identified by the numbers of the sectors occupied, the letters of the layers involved, and the vertebrae involved in the longitudinal extent of the tumor. Chemotherapy and local irradiation (45-60 Gy) were administered to two patients with osteosarcoma, and irradiation was performed on two patients (chordoma and recurrent giant cell tumor). All the specimens were submitted for histological study of the margins. Spinal pain was assessed by the scales proposed by Denis et $a l^{3}$ (Table 2). Preoperative and follow-up neurological assessments were made according to the modified Frankel grading system, supplemented by manual muscle tests and bladder function assessments. ${ }^{4}$ The patients were followed every other month for 2.0-4.8 (average, 3.2) years. Local recurrence was evaluated by plain X-rays and $\mathrm{CT}$ scans.

\section{Surgical procedure}

Detailed surgical planning was made in each case based on plain X-rays and MR images or CT scan. In principle, total spondylectomy was performed through the posterior approach alone for single level lesions at the T1 to L2 level (Figure 2A,B) and the combined single stage anterior and posterior approach for lesions at the L3 to L5 level.

Posterior total spondylectomy With the posterior approach, the patient was placed in a prone position. The laminae of the affected lumbar vertebra and two levels above and below were exposed through a posterior midline incision. The transverse processes of the affected lumbar vertebra and one level above and below on both sides were also exposed to the lateral tips using electrocautery. Biopsy or previous surgical scars were excised, taking care not to open the fibrous capsule of the tumor. The inferior half of the upper adjacent lamina, including part of the respective spinous process and the inferior facet joints, were resected to that the spinal canal was well exposed. A fine threadwire saw $0.54 \mathrm{~mm}$ in diameter designed by Tomita $e t a l^{5}$ was inserted into the sublaminar space of the affected vertebra from the exposed cephalad epidural space and pulled out from the outside of the foramen and then passed under the transverse process of the affected vertebra. The other end of the saw was passed under the superior articular process of the affected vertebra. Then both ends were pulled in the superior-lateral direction and both pedicles cut. Now

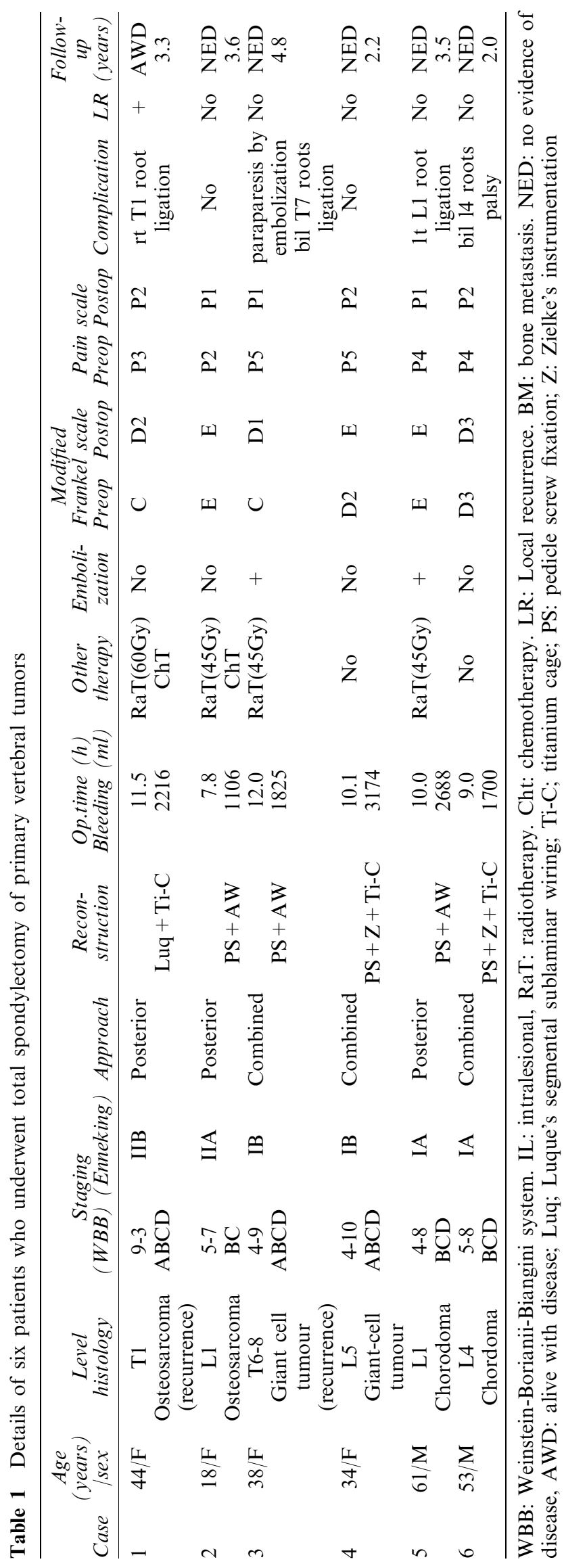


Table 2 Denis pain scale

\begin{tabular}{ll}
\hline Grade & Criteria \\
\hline P1 & No pain \\
P2 & $\begin{array}{l}\text { Occasional, minimal pain; no need for medication } \\
\text { P3 }\end{array}$ \\
Moderate pain, occasional pain, no interruption of \\
Pork or ADLs
\end{tabular}

From Denis et al. $.^{3} \mathrm{ADL}=$ activities of daily living

the posterior component was mobile and could be removed en bloc after cutting the ligamentum flavum and the inferior joint capsules. If the unilateral pedicle was affected by the tumor, osteotomy for en bloc resection was performed through the neighboring healthy lamina. If there was tumor invasion into both pedicle and lamina, osteotomy outside the tumor capsule was impossible without sacrifice of the involved nerve root, which would seriously affect radical resection, the involved nerve root was ligated and cut, and the dura was pulled to the healthy side. Then a knife or a needle of electric cautery was inserted into the affected pedicle coagulated tumor tissue inside the pedicle before pediculotomy, and the cutting surface of the pedicle was blocked with bone wax immediately after osteotomy.

In the thoracic spine, the ribs of the affected vertebra and those adjacent to them were cut off $4 \mathrm{~cm}$ lateral from the tips of the costtransverse process on the dominant side of the tumor and $2 \mathrm{~cm}$ lateral on the less dominant side. After the peritoneum and muscles had been carefully removed from the ribs, avoiding rupture of the parietal pleura, the ribs were separated from the costtransverse processes by cutting through the costtransverse ligaments. Then bilateral pediculotomy was performed in the same way as the procedure for the lumbar lesion.

En bloc resection of the anterior column After the removal of the posterior elements, meticulous coagulation of the epidural vessels was performed by bipolar diathermy. In the thoracic spine, the intercostal vessels were separated from the intercostal nerves and the branches that entered into the nerve roots and the neural foramen were cauterized. The intercostal vessels and the parietal pleura were then pushed aside from the lateral and anterior aspect of the vertebra manually or with small pledgets. The curved vertebral retractors were inserted to meet each other anterior to the vertebrae from both sides of the thoracic spine to protect the surrounding soft tissues during isolation of the affected vertebra and reconstruction of the anterior column. The affected vertebra was isolated by cutting through the upper and lower discs with two threadwire saws introduced in front of the vertebral body. Before complete separation of the affected vertebral body, the spinal column needed to be stabilized with a unilateral instrument. The affected vertebra was freed and removed by rotating it around the spinal cord. When it was difficult to roll out the separated vertebral body, sacrifice of a unilateral root was performed. In the thoracolumbar junction or the lumbar spine above the L2, the procedure was almost the same as for the thoracic spine (Figure 2A,B). The

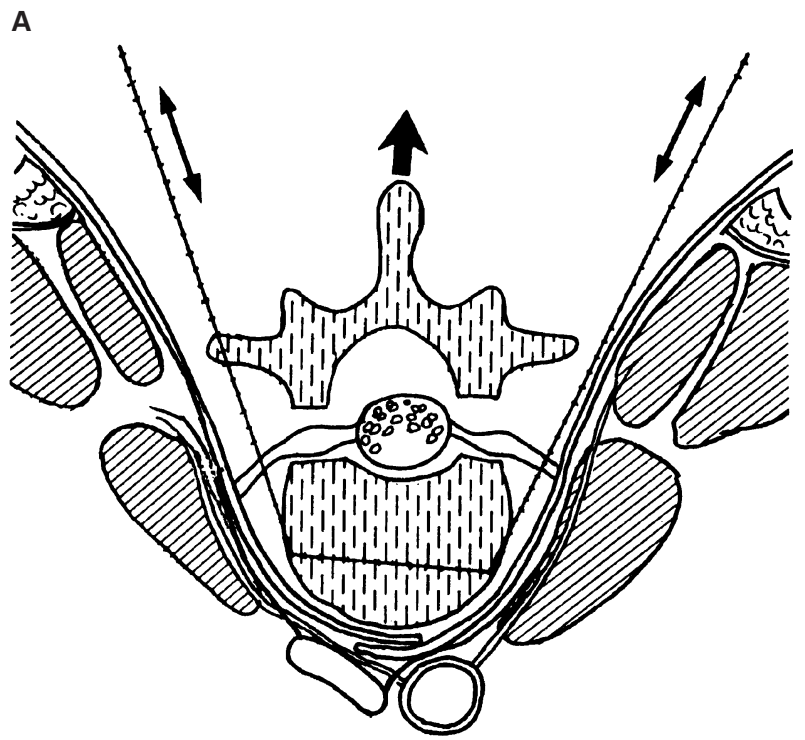

B

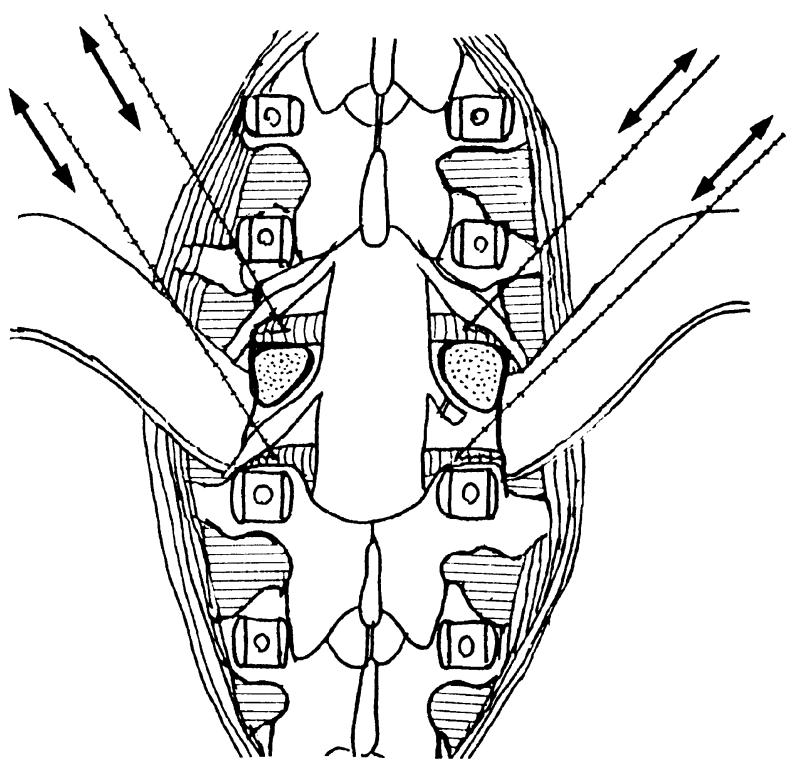

Figure 2 Posterior total spondylectomy. After en bloc resection of the neural arch by bilateral pediculotomy, en bloc corporectomy is performed at the upper and lower adjacent disc of the affected vertebra using a threadwire saw. The nerve root is cut on one side to pull out the separated vertebral body. (A) Transaxial plane, (B) Posterior view after removal of the neural arch 
psoas muscles with segmental vessels were also dissected with the finger tips or by packing sponges from the vertebral body.

Reconstruction In the thoracic spine or the lumbar spine above the L2, pedicle screws of the CotrelDubousset (CD) system were placed two vertebrae above and below through the single posterior approach. Before complete separation of the affected vertebral body, the spinal column was fixed in the in situ position by a unilateral rod to restore the normal spinal curve. Reconstruction was carried out using an apatite-wollastonite glass ceramic (AWGC) prosthesis (Lederle, Tokyo, Japan) or a titanium mesh cylinder (MOSS $^{\circledR}$ Miami, DePuy Motech, Warsaw, IN, USA). The CD rod was readjusted to fix the prosthesis firmly. Strut and cancellous bone grafts from the resected ribs, ileum and fibula were placed around the prosthesis and rods. Rotational stability was enforced with two crosslink transverse fixators.

\section{Combined single stage total spondylectomy}

Total spondylectomy of the L3, L4 and L5 vertebrae was performed by the single-stage combined anterior and posterior approaches. The fusion area was one vertebral level above and one below the affected vertebra.

The first step was a posterior approach. En bloc resection of all the posterior elements, including the transverse process through the bilateral pedicles, was performed with the threadwire saw. This procedure is the same as that of simple posterior total spondylectomy. After en bloc resection of the posterior elements and meticulous coagulation of the epidural vessels by bipolar diathermy, the surgeon went as far forward as possible in order to ease the anterior en bloc resection of the affected vertebral body, especially the side contralateral to the anterior approach. The posterior half and contralateral side of the disc adjacent above and below the affected vertebrae were cut. The spine was then stabilized using a pedicle screw system between the vertebrae above and below the affected spine. Bisected fibula graft was performed between the adjacent laminae.
The second step was performed on the same day using a retroperitoneal approach. En bloc resection of the affected vertebral body was performed by excision of the residural adjacent discs, after the entire body of the affected vertebrae was freed from the surrounding psoas muscles, segmental vessels and soft tissue. Anterior spinal reconstruction was performed using AWGC spacer or titanium cage packed bone chips of iliac bone and Zielke's anterior spinal instrumentation.

Postoperative management One week later when the neurological and general condition of the patient had been restored, the patient was allowed out of bed and was ambulatory. Patients with lumbar lesions wore a fabric corset until adequate protective muscle tone was attained.

\section{Results}

Table 1 provides details on each patient. All the vertebrae with tumors were resected. Anterior fusion was carried out at two intervertebral levels in five patients (cases 1, 2, 4, 5, and 6) and at four intervertebral levels in one patient (case 3). AWGC vertebral prostheses were used in three patients and titanium mesh cylinders in three. For all patients, treatment was augmented with autogenous bone grafting (fibulas, ribs and/or iliac bones). Anterior instrumentation was performed in two cases with two intervertebral fusions in the lumbar spine through the combined approach. The extent of posterior stabilization was determined by the principle of 'two above, two below' with posterior instrumentation in four patients, and 'one above, one below' with posterior and anterior instrumentation in two patients. The duration of surgery ranged from 7.8 to 12.0 (mean, 10.1) h. Blood loss varied from 1106 to 3174 (mean, 2118) g. Both parameters increased following one-stage total spondylectomy through the combined approach. There were neither fatal complications nor bleeding that was difficult to control from the segmental or the other large vessels. Histological assessment of the surgical margin of the resected specimens is shown in Table 3.

The final follow-up study was carried out 2.0 to 4.8 (mean, 3.2) years after surgery; one patient was alive with disease, the other five patients were alive without

Table 3 Histological assessment of the surgical margins of the resected specimen

\begin{tabular}{|c|c|c|c|c|c|c|c|c|c|}
\hline \multirow[b]{2}{*}{ Case } & \multirow[b]{2}{*}{$\begin{array}{l}\text { Vertebral } \\
\text { body }\end{array}$} & \multirow[b]{2}{*}{$\begin{array}{l}\text { Pedi- } \\
\text { cle }\end{array}$} & \multicolumn{2}{|c|}{ Horizontal plane } & \multirow[b]{2}{*}{$\begin{array}{c}\text { Spinal } \\
\text { canal }\end{array}$} & \multicolumn{2}{|c|}{ Vertical plane } & \multirow[b]{2}{*}{$\begin{array}{l}\text { Local } \\
\text { recurrence }\end{array}$} & \multirow[b]{2}{*}{$\begin{array}{c}\text { Final } \\
\text { follow-up (years }\end{array}$} \\
\hline & & & $\begin{array}{c}\text { Transverse } \\
\text { process }\end{array}$ & $\begin{array}{l}\text { Lamina and } \\
\text { spinous process }\end{array}$ & & $\begin{array}{l}\text { Upper } \\
\text { end }\end{array}$ & $\begin{array}{l}\text { Lower } \\
\text { end }\end{array}$ & & \\
\hline 1 & W & $\mathrm{Mc}$ & I & Mc & $\mathrm{Mc}$ & W & W & + & 3.3 \\
\hline 2 & W & $\mathrm{W}$ & W & $\mathrm{W}$ & $\mathrm{W}$ & W & W & No & 3.6 \\
\hline 3 & M & $\mathrm{W}$ & W & $\mathrm{W}$ & M & W & W & No & 4.8 \\
\hline 4 & $\mathrm{M}$ & Mc & M & Mc & $\mathrm{Mc}$ & W & W & No & 2.2 \\
\hline 5 & W & Mc & W & W & $\mathrm{M}$ & $\mathrm{M}$ & M & No & 3.5 \\
\hline 6 & W & $\mathrm{W}$ & $\mathrm{W}$ & W & M & M & $\mathrm{W}$ & No & 3.2 \\
\hline
\end{tabular}

$\mathrm{W}$ : wide margin; M: marginal margin; I intralesional margin; Mc: marginal margin with possible contamination 
evidence of disease. Local recurrence was detected in patient 1, who had showed osteosarcoma of the T1 posterior column after intralesional decompression laminectomy, resulting in an intralesional margin at the right costtransverse process. Two patients with P5 by Denis' pain score ${ }^{3}$ before surgery achieved levels of $\mathrm{P} 1$ or $\mathrm{P} 2$ after surgery. $\mathrm{P} 4$ changed to $\mathrm{P} 2$ in two cases and $\mathrm{P} 1$ in one case. P1 was not changed by surgery in one case. Four of the five patients with neurological involvement showed at least a one-grade improvement on the modified Frankel scale. ${ }^{4}$ Two patients with grade $\mathrm{C}$ achieved grades $\mathrm{D} 1$ and $\mathrm{D} 2$. Two patients with grade $\mathrm{E}$ and one with D3 did not change. One with grade D2 achieved grade E (Table 1).

All the grafted bones fused completely without instrument failure, major dislodgment of bone grafts or artificial vertebral implants at follow-up examination. Sacrifice of the nerve roots of the affected vertebra was necessary in three cases. There were neurological complications for two patients. In case 3, neurological disturbance worsened after preoperative embolization and permanent paraparesis occurred. Patient 6 exhibited transient nerve root palsy caused by laceration of the nerve root sheath when the successfully isolated L4 vertebral body was rolled away from the dural tube between the L4 and L5 nerve root.

\section{Discussion}

Two types of total spondylectomy are described in the literature, piecemeal resection ${ }^{6-8}$ and en bloc resection, which is more demanding technically. ${ }^{2,5,9-11}$ Piecemeal total spondylectomy was performed in case 3 at the initial surgery, but it has not prevented local recurrence, even though the tumor was benign aggressive. Intralesional piecemeal total spondylectomy is apt to cause massive bleeding that is difficult to control and to result in a less radical excision and in higher contamination by the tumor cells. En bloc resection, even if marginal, seems to be more effective treatment in local control of spinal tumors, especially in chordoma. ${ }^{12}$ Tomita et al reported good results for their 'total en bloc spondylectomy', which occasionally resulted in an intralesional margin or marginal margin with contamination at the pedicle or the spinal canal, although their follow-up study was not long enough to evaluate local recurrence. ${ }^{5,13}$

Total spondylectomy with en bloc resection of the tumor is considered to be a good indication for patients with malignant or aggressive benign tumors satisfying the following criteria: the tumor has not spread into or invaded adjacent visceral organs, including the intradural area, vena cava or aorta, the tumor does not show multiple metastasis, and the tumor lesion has contiguous involvement of fewer than three vertebrae. Radical excision after a previous intralesional excision is supposed to be worthwhile for giant cell tumors (case 3), but not for osteosarcoma (case 1), nor possibly chordoma. According to Fielder, ${ }^{11}$ successful radical resection has two prerequisites. First, part of the neural arch and the underlying epidural space must be normal, so that the bony ring around the dura can be broached through healthy tissue and allow for the diseased vertebral segment to be rolled away from the dural tube. Secondly, it must be possible to divide any involved nerve root at its junction with the dura without overhanging tumor leading to possible contamination of the operation site. However, these cases are very rare (four cases among 21 cases of chordoma, Boriani; ${ }^{12}$ none among seven cases of primary malignant tumor, Tomita et $a l^{13}$ ). In the present study, there were only two of six cases that satisfied this condition.

There is controversy over which surgical approach to take for total spondylectomy, the posterior approach or anterior and posterior combined approach. Roy-Camille et al recommended that the origins of psoas muscles from the lumbar spine make single-stage posterior total spondylectomy unfeasible. ${ }^{14}$ Because of the close proximity of major abdominal vessels to the anterior vertebral column in the lumbar spine, they recommended a two-stage operation to resect malignant vertebral neoplasmas occurring between L1 and L4. Stener reported total spondylectomy through a single-stage posterior approach for tumors at L3 or the cephalad. ${ }^{15} \mathrm{He}$ advocated the combined anteroposterior approach for tumors at L4. Tomita et al reported that posterior total en bloc spondylectomy was possible even at L4. ${ }^{5}$ We consider the posterior approach should be used for lesions from levels T1 to L2 if there is not the considerable tumor extension outside the vertebral body involving segmental vessels impossible to separate outside the tumor capsule. And for the middle or lower lumbar spine and for the lesion with the massive tumor extension outside the vertebral body at any level, the combined single stage anterior and posterior approach for the following four reasons. First, the size of the resected vertebral body is large enough to injure the nerve roots or the lumbar nerve plexus when it is rolled out around the dural tube between the nerve roots without cutting the nerve root, as in case 6 . Second, serious neurological deficits resulted from sacrifice of the L3, L4 or L5 nerve. Third, the lateral exposure of the spine is likely to be limited by the iliac crests at L4 or L5. Fourth, it is important to preserve the motion segment in the lower lumbar spine by minimizing segmental fusion. A combination of anterior and posterior instrumentation saves the number of motion segments to be sacrificed for spinal reconstruction, compared to posterior instrumentation only. ${ }^{16}$

Although the authors have not encountered this, the most serious risk of posterior total spondylectomy is considered to be severe anterior hemorrhage during isolation of the vertebral body. Maintenance of close vertebral body or tumor capsule contact by a gentle dissection technique throughout the exposure and step-wise progression with meticulous hemostasis is 
supposed to be important. The second most serious risk seems to be insufficiency of blood supply to the spinal cord, although we have not experienced this. Hodgson et al reported there was no circulatory trouble in the spinal cord, no matter how many segmental arteries were divided unilaterally, ${ }^{17}$ and Tomita et al reported that there were not serious circulatory complications involving the spinal cord in their bilateral division of the spinal branches of the segmental vessels in the three maximal contiguous segments. ${ }^{5,13}$

Although the present report should be defined as a preliminary one concerning the oncological results, some interesting results have been obtained. First, the surgical invasion and complication rate for this difficult resection were considered acceptable. Second, despite the limitations of en bloc resection outside of the tumor capsule, which are that the surgical margin was marginal, and that contamination was possible in a considerable number of cases in which intralesional invasion was minimized as much as possible, this procedure is considered to be better in terms of radicality and in local control of tumor recurrence than piecemeal total spondylectomy in cases of lowgrade malignancy or aggressive benign tumors.

\section{References}

1 Enneking WF, Spanier SS, Goodmann MA. A system for the surgical staging of musculoskeletal sarcoma. Clin Orthop 1980; 153: $106-120$.

2 Boriani $\mathrm{S}$ et al. En bloc resection of bone tumors of the thoracolumbar spine: A preliminary report on 29 patients. Spine 1996; 21: $1927-1931$.

3 Denis F, Armstrong GWD, Seals K, Matta L. Acute thoracolumbar burst fractures in the absence of neurological deficit: A comparison between operative and non-operative treatment. Clin Orthop 1984; 189: 142-149.
4 Bradford DS, McBride GG. Surgical management of thoracolumbar spine fractures with incomplete neurologic deficits. Clin Orthop 1987; 218: $201-216$.

5 Tomita $\mathrm{K}$ et al. Total en bloc spondylectomy for solitary spinal metastasis. Int Orthop 1994; 18: 291-298.

6 Liévre JA et al. Tumeur á cellules géantes du rachis lombaire, spondylectomie totale en deux temps. Rev Rhum 1968; 35: $125-$ 130.

7 Magerl F, Corcia MF. Total posterior vertebrectomy of the thoracic or lumbar spine. Clin Orthop 1988; 232: 62-69.

8 Sundaresan N, DiGiacinto GV, Hughes JEO, Krol G. Spondylectomy for malignant tumors of the spine. J Clin Oncol 1989; 7: $1485-1491$.

9 Stener B. Total spondylectomy for removal of a giant-cell tumor in the eleventh thoracic vertebra. Spine 1977; 2: 197-201.

10 Stener B. Complete removal of vertebrae for extirpation of tumors: A 20-year experience. Clin Orthop 1989; 245: $72-82$.

11 Fidler MW. Radical resection of vertebral body tumors: A surgical technique used in ten cases. J Bone Joint Surg [Br] 1994; 76-B: $765-772$.

12 Boriani $\mathrm{S}$ et al. Chordoma of the spine above the sacrum: Treatment and outcome in 21 cases. Spine 1996; 13: 1569-1577.

13 Tomita $\mathrm{K}$ et al. Total en bloc spondylectomy: A new surgical technique for primary malignant vertebral tumors. Spine 1997; 22: $324-333$.

14 Roy-Camille R, Mazel Ch, Saillant G, Lapresle PH. Treatment of malignant tumors of the spine with posterior instrumentation. In: Sundaresen N, Schmidek HH, Schiller AL, Rosenthal DI (ed). Tumor of the Spine Philadelphia: WB Saunders; 1990, pp $473-$ 487.

15 Stener B. Technique of complete spondylectomy in the thoracic and lumbar spine. In: Sundaresen N, Schmidek HH, Schiller AL, Rosenthal DI (ed). Tumor of the Spine. Philadelphia: WB Saunders; 1990, pp $432-437$.

16 Oda I et al. The stability of reconstruction methods after thoracolumbar total spondylectomy: An in vitro investigation. Spine 1999; 24: $1634-1638$.

17 Hodgson AR, Stock FE, Fang HSY, Ong GB. Anterior spinal fusion: the operative approach and pathological findings in 412 patients with Pott's disease of the spine. Br J Surg 1960-1961; 48: $172-178$. 